\title{
Autologous LMP1-/LMP2- Specific Cytotoxic T-Lymphocytes
}

National Cancer Institute

\section{Source}

National Cancer Institute. Autologous LMP1-/LMP2-Specific Cytotoxic T-Lymphocytes. NCl Thesaurus. Code C70836.

A preparation of cytotoxic T-lymphocytes (CTL), specifically reactive to the Epstein-Barr virus (EBV) latent membrane proteins (LMP) 1 and 2, with potential antineoplastic activity. Autologous dendritic cells and EBV-infected lymphoblastoid cell lines (LCL) from patients with EBV-positive nasopharyngeal carcinoma (NPC) are transduced with an LMP1/LMP2expressing adenoviral vector, are irradiated, and then are used to stimulate and expand autologous CTL to produce autologous LMP1-/LMP2-specific CT L ex vivo.

Administration of autologous LMP1-/LMP2- specific cytotoxic T-lymphocytes may result in a specific CTL response against tumor cells expressing LMP1 and LMP2, resulting in cell lysis and inhibition of tumor cell proliferation in vivo. Among a limited set of viral antigens expressed by NPC cells, LMP1 and LMP2 are weak immunogens which, nevertheless, are capable of inducing a T-lymphocyte response. 